\title{
Virtual Reference Feedback Tuning with bayesian regularization
}

\author{
Gianmarco Rallo $^{+}$, Simone Formentin ${ }^{+}$, Alessandro Chiuso*, Sergio M. Savaresi ${ }^{+}$
}

\begin{abstract}
Virtual Reference Feedback Tuning (VRFT) is a well established tool to design model-reference controllers directly from input-output data. A major drawback of the method lies in that the variance of the controller is high, due to the instrumental variable method employed to obtain unbiased estimates. Recent results on the use of kernel-based regularization in system identification showed that a good biasvariance trade-off can be found by suitably tuning a penalty term in the identification criterion within a Bayesian framework. In this paper, we apply such a regularization approach to the VRFT method and we show that significant performance improvement can be obtained also for controller design. A benchmark example is used to illustrate the effectiveness of the proposed approach.
\end{abstract}

\section{INTRODUCTION}

In modern model-based control design, a model of the plant is first identified from data or developed from first principles and then used to design a feedback controller satisfying some closed-loop requirements, typically stability, reference tracking or disturbance rejection performance. Within this context, various controller design approaches have been proposed [6]. In fixed-order model reference control, a controller-order reduction step is also needed before implementation [1].

Unfortunately, the resulting controller is not necessarily optimal when connected to the plant, as the closed-loop performance is limited by modeling errors. Therefore, several direct data-driven controller tuning techniques have been proposed to avoid the problem of undermodeling and to facilitate the design of fixed-order controllers, both iteratively e.g. [12], [13] and non-iteratively e.g. [2], [23]. In all the above approaches, only the final control cost is taken into account and the controller is directly obtained from data, without first deriving a model of the system.

Among all, the Virtual Reference Feedback Tuning (VRFT [2]) method is a noniterative approach, which is based on simple least squares formulas and instrumental variables. Within this framework, linear-in-the-parameters controllers, like PIDs, can be rapidly designed and recalibrated with new experiments.

Various examples have shown the effectiveness of the VRFT approach in simulation and real-world applications, see e.g. [7], [8]. However, since the method is based on

+ Dipartimento di Elettronica, Informazione e Bioingegneria, Politecnico di Milano, via Ponzio 34/5, 20133 Milano (Italy). Email to: name. surname@polimi.it.

* Department of Information Engineering, University of Padova, Via Gradenigo 6/b, 35131 Padova, Italy. Email to: chiuso@dei.unipd.it. errors-in-variables estimation [21], it is not statistically efficient, i.e. the Cramér-Rao lower bound cannot be achieved [24].

To enhance the statistical performance of the methods, it has been shown in [10] that the VRFT design can be reformulated as an $\mathcal{L}_{2}$-regularized optimization problem. By suitably tuning the $\mathcal{L}_{2}$ penalty term in the cost function, the quality of the resulting controller can be significantly improved, as $\mathcal{L}_{2}$-regularization is very suited to cope with high variance estimation problems [5]. The regularization term of [10] is tuned based on data-driven convex-concave procedures, whereas the a-priori knowledge of the system cannot be exploited.

A different perspective in regularization can be followed, according to the Bayesian point of view introduced for system identification in [18] and its follow-up (see, e.g. [17]). Specifically, the idea of the above papers is to recast the identification of the impulse response of a system into an infinitedimensional space learning problem, instead of considering finite-dimensional parameterization. Within this framework, regularization techniques correspond to impose certain prior distributions on the impulse response parameters, thus further enhancing the quality of the estimate. To the best of the authors' knowledge, the same philosophy will be applied in this work for the first time to data-driven controller design.

Then, the aim of this paper is two-fold: (i) to apply the Bayesian approach in [18] to tune the kernel of regularized VRFT and use priors on the system also for controller identification, (ii) to show that such a Bayesian perspective may outperform the existing regularized VRFT introduced in [10].

The outline of the paper is as follows. In Section II, the VRFT method is briefly recalled. In Section III, the regularized version of VRFT is introduced in the Bayesian framework, where the main tuning parameters are presented and discussed. The optimal regularization term is derived in Section IV, to obtain the best achievable performance. The regularized methods will be compared to standard VRFT and to the method [10] on the benchmark example [14] in Section $\mathrm{V}$. The paper is ended by some concluding remarks.

\section{THE VRFT METHOD}

Consider the unknown LTI SISO stable plant $G\left(q^{-1}\right)$, where $q^{-1}$ denotes the backward shift operator. The control problem considered in this paper is to design a linear, fixed-order controller $K\left(q^{-1}, \rho\right)$, parameterized through $\rho$, for which the closed-loop system matches a user-defined reference model $M\left(q^{-1}\right)$. 
More specifically, we consider a linear controller parameterization

$$
K\left(q^{-1}, \rho\right)=\beta^{T}\left(q^{-1}\right) \rho,
$$

where $\beta\left(q^{-1}\right)$ is a vector of $n$ linear discrete-time transfer operators, each one defined as

$$
\beta_{i}\left(q^{-1}\right)=\frac{q^{-i}}{1-q^{-1}}, \quad i=0, \ldots, n-1 .
$$

More formally, our aim is to find $\rho$ minimizing the (filtered) $\mathcal{H}_{2}$-norm of the difference between the reference model and the closed-loop system achieved with $K\left(q^{-1}, \rho\right)$, namely

$$
J_{m r}(\rho)=\left\|\left(\frac{G K(\rho)}{1+G K(\rho)}-M\right) W\right\|_{2}^{2},
$$

where $W\left(q^{-1}\right)$ is a user-defined frequency-weighting filter.

The goal can be interpreted as to find the minimizer of the $\mathcal{L}_{2}$-norm of the matching error signal $\varepsilon$ in Figure 1, when the reference signal is a white noise of unit variance. From now on, under the assumption that $M$ is achievable, i.e. if there exists a controller in the considered controller class such that $M$ is exactly obtained in closed-loop, the minimizer of (3) will be referred to as "optimal controller" and will be denoted by $K_{o}\left(q^{-1}\right)=K\left(q^{-1}, \rho_{o}\right)=\beta^{T}\left(q^{-1}\right) \rho_{o}$.

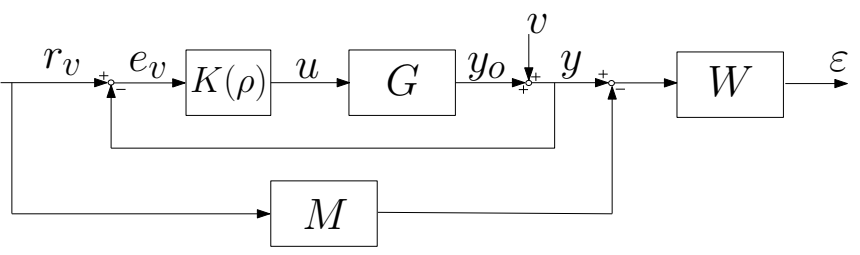

Fig. 1. Model reference control problem.

Consider now that an open-loop collection of input-output (I/O) data $\{u(t), y(t)\}_{t=1, \ldots, N}$ collected from the system is available and let the output $y(t)$ be affected by a zero mean white noise $v(t)$ of variance $\sigma^{2}$.

In standard model-based approaches, the above objective can be achieved by identifying from data a model $\hat{G}$ of the plant and designing a model-based controller $K(\hat{G})$ as

$$
\hat{K}=\frac{M}{\hat{G}(1-M)} .
$$

In this work, the case where the controller is directly derived from the data collection without identifying $\hat{G}$ is instead considered. Notice that in the model-based approach, although $M$ is supposed here to be achievable, $K_{o}$ can be derived from (4) only when $\hat{G}=G$, i.e. when there are no modeling errors.

The idea of Virtual Reference Feedback tuning was first proposed in [11] with the name of Virtual Reference Direct Design $\left(V R D^{2}\right)$ and subsequently fixed and extended in [2], [3] and [9] respectively for LTI, nonlinear and LPV systems.

The main idea to minimize (3) without identifying $G\left(q^{-1}\right)$ is to build a "virtual" closed-loop system, where the input and output signals are equal to $u(t)$ and $y(t)$ and the closedloop transfer function is assumed to correspond to $M\left(q^{-1}\right)$. From such loop, the so-called "virtual reference" $r_{v}(t)$ and "virtual error" $e_{v}(t)$ signals can be computed as

$$
r_{v}(t)=M^{-1}\left(q^{-1}\right) y(t), e_{v}(t)=r_{v}(t)-y(t),
$$

as illustrated in Figure 2. The control design issue is then

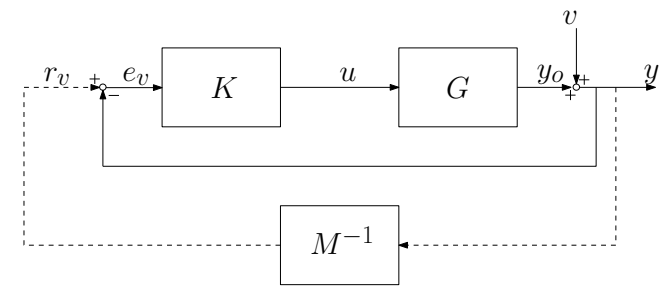

Fig. 2. The VRFT rationale for the calculation of the "virtual" signals.

reduced to an identification problem, where the optimal controller is the one that generates $u(t)$ when fed by $e_{v}(t)$. The criterion to be minimized is then

$$
J_{v r}^{N}(\rho)=\frac{1}{N} \sum_{t=1}^{N}\left(u_{L}(t)-K\left(q^{-1}, \rho\right) e_{L}(t)\right)^{2},
$$

where $u_{L}(t)=L\left(q^{-1}\right) u(t), e_{L}(t)=L\left(q^{-1}\right) e_{v}(t)$ and $L\left(q^{-1}\right)$ is a suitable prefilter such that (5) is equal to the second-order Taylor expansion of (3) in the neighborhood of the minimum point [2]. More specifically, the frequency response of $L\left(q^{-1}\right)$ must be such that

$$
L\left(e^{-j \omega}\right)=\frac{M\left(e^{-j \omega}\right)\left(1-M\left(e^{-j \omega}\right)\right) W\left(e^{-j \omega}\right)}{\Phi_{u}^{1 / 2}(\omega)},
$$

where $\Phi_{u}^{1 / 2}(\omega)$ denotes a spectral factor of $\Phi_{u}(\omega)$. For the final estimate not to be biased, basic instrumental variables (IV) [15] are used to counteract the effect of noise.

The final formula to find the optimal controller reads

$$
\hat{\rho}_{N}^{\mathrm{IV}}=R_{N}^{-1} F_{N}
$$

where

$$
R_{N}=\frac{1}{N} \sum_{t=1}^{N} \varphi_{2}(t) \varphi^{T}(t), F_{N}=\frac{1}{N} \sum_{t=1}^{N} \varphi_{2}(t) u(t)
$$

and

$$
\begin{aligned}
\varphi(t) & =\beta\left(M^{-1}-1\right) L y(t) \\
& =\beta\left(M^{-1}-1\right) L G u(t)+\beta\left(M^{-1}-1\right) L v(t) \\
& =\varphi_{o}(t)+\tilde{\varphi}(t) \\
\varphi_{2}(t) & =\beta\left(M^{-1}-1\right) L y_{2}(t)
\end{aligned}
$$

being $y_{2}(t)$ the output of a second experiment on the plant $G$. Since $M$ is assumed to be achievable with the given controller parameterization, we have $u(t)=\varphi_{o}^{T}(t) \rho_{o}$. According to the theory in [2], the second experiment must be performed using the same input sequence $u(t)$ of the first one, such that the only difference between $y(t)$ and $y_{2}(t)$ is a different realization of the noise $v(t)$. 


\section{BAYESIAN REGULARIZATION}

In its classical formulation, the VRFT method provides a consistent estimate of the optimal controller, that means that, as $N$ goes to infinity, the minimum of the data-driven criterion coincides with $K_{o}$. Since the method relies on IV techniques to cope with measurement noise, the variance of the parameter estimate is much larger than the Cramér-Rao lower bound [24]. However, since the final goal in practice is the minimization of (3) for a given $N$ and not to find an unbiased controller, the bias and variance effects can be balanced via suitable trade-off tuning.

The problem can be faced from a Bayesian perspective, analogously to what has been recently done in system identification [20]. In particular, the works presented in [18] and [17] show that the identification of the impulse response of stable systems can be reinterpreted as a function learning problem in a space of function, called Reproducing Kernel Hilbert Space (RKHS), which is endowed with particular smoothness properties.

In our case, the controller parameterization in (1) can be rewritten as

$$
K\left(q^{-1}, \rho\right)=\frac{1}{1-q^{-1}} \sum_{i=0}^{n-1} \rho_{i} q^{-i}=\frac{1}{1-q^{-1}} \bar{K}\left(q^{-1}, \rho\right),
$$

with an obvious definition for $\bar{K}\left(q^{-1}, \rho\right)$. Therefore, the problem can be seen as a FIR identification problem, where the (maybe high order) FIR to be identified is $\bar{K}\left(q^{-1}, \rho\right)$ and the $\rho_{i}$ 's represent the Markov parameters of the controller (without the integral part).

In a Bayesian framework, such parameters can be seen as the realization of a gaussian process with zero mean and covariance matrix $\Pi$, namely

$$
\rho \sim \mathcal{N}(0, \Pi),
$$

where $\Pi(t, \tau)=\mathbb{E}\left[\rho_{t} \rho_{\tau}\right]$ is called kernel from now on. Selecting the shape of such a kernel allows to define the prior knowledge about the controller, i.e. $\rho$.

In previous works (see [20] and references therein), it has been shown that the Bayesian estimate of $\rho$ corresponds to minimizing the regularized cost function

$$
\hat{\rho}_{N}=\arg \min _{\rho}\left[J_{v r}^{N}(\rho)+\rho^{T} D \rho\right],
$$

where $D \in \mathcal{R}^{n \times n}$ is a matrix to be tuned based on the knowledge (or tuning) of the kernel $\Pi$ as

$$
D^{-1}=\frac{\Pi}{\sigma^{2}} \text {. }
$$

Notice that (13) generally provides a biased estimate of $K_{o}$.

The problem is how to parameterize and design $\Pi$. In case of stable optimal controller (it is always the case in well-posed model reference control problems), the variance of $\rho_{t}$ is expected to tend to zero exponentially, with a certain decay rate $\alpha$, because the impulse response of a stable system annihilates for large time $t$. Moreover, if the impulse response is supposed to be smooth, the neighbour coefficients have positive correlation. From these two hypotheses, the so called Stable Spline Kernel can be built as

$$
\Pi_{S S}(t, \tau)=\lambda\left(\frac{e^{-\alpha(t+\tau+\max [t, \tau])}}{2}-\frac{e^{-3 \alpha \max [t, \tau]}}{6}\right),
$$

where $\lambda$ is a scaling factor.

The structure of $\Pi_{S S}$ is defined as a function of some hyperparameters, specifically $\alpha$ and $\lambda$. This formulation transforms the problem of estimation of a (potentially infinite) number of FIR coefficients into the estimate of a limited number of variables concerning the correlation among the coefficients.

Such hyperparameters can be estimated according to the empirical Bayes approach [20], which relies on the marginal likelihood maximization of the data with respect to $\alpha, \lambda$.

Other forms of the kernel matrix can be considered. For istance, if the impulse response of the controller is non-smooth and rapidly varying, it is necessary to include the case of negative correlation between impulse response coefficients. In this case, a suitable choice of the kernel can be the High Frequency Stable Spline defined as

$$
\Pi_{H F}(t, \tau)=\lambda \begin{cases}\max \left(e^{-\alpha t}, e^{-\alpha \tau}\right) & \text { if } t+\tau \text { is even } \\ -\max \left(e^{-\alpha t}, e^{-\alpha \tau}\right) & \text { if } t+\tau \text { is odd. }\end{cases}
$$

Another kernel is the Diagonal correlated

$$
\Pi_{D C}(t, \tau)=\lambda \epsilon^{(t+\tau) / 2} \theta^{|t-\tau|},
$$

where $\lambda \geq 0$ is the scale factor, $0 \leq \epsilon \leq 1$ accounts for the exponential decay rate along the diagonal, and $|\theta| \leq 1$ describes the correlation across the diagonal. The tuned correlated kernel $T C$ is a particular case of $D C$ kernel, where $\theta=\sqrt{\epsilon}$

$$
\Pi_{T C}(t, \tau)=\lambda \epsilon^{\max (t, \tau)} .
$$

Finally, $D I$ is a simple diagonal kernel

$$
\Pi_{D I}(t, \tau)=\lambda \operatorname{diag}\left(\mu_{0}, \ldots, \mu_{n-1}\right) .
$$

In the latter case, there are no particular hypotheses on the impulse response function to estimate and every element of the matrix $\mu_{i}$ has to be evaluated as a hyperparameter. A more detailed analysis of the available kernel choices is provided in [19] and [4].

\section{OPTIMAL KERNEL SELECTION}

In this section, the ideal kernel for VRFT is found, following the analogous result for system identification in [5], as the one minimizing the Mean Square Error of the estimate. This kernel will constitute an upper bound for the achievable performance and will be a useful tool to evaluate the estimates with the different kernels.

The regularized VRFT estimate reads

$$
\hat{\rho}_{N}^{\mathrm{rIV}}=\left(R_{N}+D\right)^{-1} F_{N}=\left(R_{N}+D\right)^{-1} R_{N} \hat{\rho}_{N}^{\mathrm{IV}} .
$$

Consider the accuracy of the standard IV estimate as presented in [22]. Under the hypothesis that optimal controller 
$K_{o}$ belongs to the considered class, the instrumental variable based estimate is such that

$$
\mathbb{E}\left[\hat{\rho}_{N}^{I V}\right]=\rho_{o}
$$

and the asymptotic covariance matrix is given by

$$
\mathbb{E}\left[\left(\hat{\rho}_{N}^{\mathrm{IV}}-\rho_{o}\right)\left(\hat{\rho}_{N}^{\mathrm{IV}}-\rho_{o}\right)^{T}\right]=\sigma^{2} R_{o}^{-1}\left(W_{1}+W_{2}\right) R_{o}^{-1},
$$

where

$$
\begin{gathered}
R_{o}=\frac{1}{N} \sum_{t=1}^{N} \varphi_{o}(t) \varphi_{o}^{T}(t) \\
W_{1}=\lim _{N \rightarrow \infty} \frac{1}{N} \sum_{t=1}^{N}\left[H^{*}\left(q^{-1}\right) \varphi_{o}(t)\right]\left[H^{*}\left(q^{-1}\right) \varphi_{o}(t)\right]^{T} \\
W_{2}=\mathbb{E}\left\{\left[H^{*}(z) \tilde{\varphi}_{2}(t)\right]\left[H^{*}(z) \tilde{\varphi}_{2}(t)\right]^{T}\right\}
\end{gathered}
$$

with $\tilde{\varphi}_{2}(t)=\varphi_{2}(t)-\varphi_{o}(t)$ and

$$
H^{*}\left(q^{-1}\right)=K_{o}\left(q^{-1}\right)\left(1-M\left(q^{-1}\right)\right)^{2} .
$$

Now, as in [5], the MSE matrix of $\hat{\rho}_{N}^{\mathrm{rIV}}$ can be calculated with respect to the true impulse response coefficients vector $\rho_{o}$. From the asymptotic value of the estimate,

$$
\mathbb{E}\left[\hat{\rho}_{N}^{\mathrm{rIV}}\right]=\left(R_{o}+D\right)^{-1} R_{o} \rho_{o},
$$

it is possible to define the bias of $\hat{\rho}_{N}^{\mathrm{rIV}}$ as

$$
\rho_{\text {bias }}^{\mathrm{rIV}}=\mathbb{E}\left[\hat{\rho}_{N}^{\mathrm{rIV}}\right]-\rho_{o}=-\left(R_{o}+D\right)^{-1} D \rho_{o} .
$$

Then, defining $\tilde{\rho}$ as

$$
\begin{aligned}
\tilde{\rho} & =\hat{\rho}_{N}^{\mathrm{rIV}}-\mathbb{E}\left[\hat{\rho}_{N}^{\mathrm{rIV}}\right] \\
& =\left(R_{N}+D\right)^{-1} R_{N} \hat{\rho}_{N}^{\mathrm{IV}}-\left(R_{o}+D\right)^{-1} R_{o} \rho_{o},
\end{aligned}
$$

the expression of the variance of the regularized estimate becomes

$$
\begin{aligned}
\mathbb{E}\left[\tilde{\rho} \tilde{\rho}^{T}\right] & =\left(R_{o}+D\right)^{-1} R_{o} \mathbb{E}\left[\left(\hat{\rho}_{N}^{\mathrm{IV}}-\rho_{o}\right)\left(\hat{\rho}_{N}^{\mathrm{IV}}-\rho_{o}\right)^{T}\right] R_{o}\left(R_{o}+D\right)^{-1} \\
& =\left(R_{o}+D\right)^{-1}\left[\sigma^{2}\left(W_{1}+W_{2}\right)\right]\left(R_{o}+D\right)^{-1}
\end{aligned}
$$

Finally, it is possible to compute the mean square error of $\hat{\rho}_{N}^{\mathrm{rIV}}$ as

$$
\begin{aligned}
& \mathrm{MSE}=\mathbb{E}\left[\tilde{\rho} \tilde{\rho}^{T}\right]+\rho_{\text {bias }}^{\mathrm{rIV}}\left(\rho_{\text {bias }}^{\mathrm{rIV}}\right)^{T} \\
& =\left(R_{o}+D\right)^{-1}\left[\sigma^{2}\left(W_{1}+W_{2}\right)+D \rho_{o} \rho_{o}^{T} D\right]\left(R_{0}+D\right)^{-1} \\
& =\left(D^{-1} R_{o}+I\right)^{-1}\left[D^{-1} W D^{-1}+\rho_{o} \rho_{o}^{T}\right]\left(D^{-1} R_{o}+I\right)^{-1}
\end{aligned}
$$

where $W=\sigma^{2}\left(W_{1}+W_{2}\right)$.

The MSE expression (29) is very similar to Equation (22) of [20], which is valid in case of additive noise on the output. Now the additive noise is on the input of the system to identify, namely the virtual error $e_{v}(t)$, which comes from the noisy output of the system $y(t)$. It follows that in (29), $W$ replaces $R_{o}$. However, the following Proposition holds.

Proposition 1: Let the MSE of the controller estimate be as in (29). The optimal kernel minimizing the MSE is given by

$$
\Pi_{o}=\rho_{o} \rho_{o}^{T}
$$

Proof: The proof follows the line of the proof of Lemma 2 in [5]. It suffices to observe that $\Pi_{o}$ makes the MSE smaller than with any other $\Pi$ also in (29), for any positive semi-definite matrix $W$.

The above results means that the optimal regularization term for controller identification (in a errors-in-variable framework) corresponds to

$$
D_{o}^{-1}=\frac{\rho_{o} \rho_{o}^{T}}{\sigma^{2}}
$$

and coincides with the one used in system identification where the input is noiseless and the output is noisy.

\section{SIMULATION EXAMPLE}

In order to evaluate the performance of the regularized VRFT control strategy, the benchmark example of [14] is considered. Let the plant be

$$
G\left(q^{-1}\right)=\frac{0.28261 q^{-3}+0.50666 q^{-4}}{A\left(q^{-1}\right)},
$$

where $A\left(q^{-1}\right)=1-1.41833 z^{-1}+1.58939 z^{-2}-$ $1.31608 z^{-3}+0.88642 z^{-4}$. Now take the feedback controller

$$
K_{o}\left(q^{-1}\right)=\frac{\sum_{k=0}^{5} \rho_{o k} q^{-k}}{1-q^{-1}}
$$

where $\rho_{o}=[0.2045 ; 0.2715 ; 0.2931 ; 0.1643 ; 0.0084]^{T}$ and consider the reference model built as

$$
M\left(q^{-1}\right)=\frac{G\left(q^{-1}\right) K_{o}\left(q^{-1}\right)}{1+G\left(q^{-1}\right) K_{o}\left(q^{-1}\right)},
$$

Notice that, by construction, $M$ is achievable if the controller class

$$
K\left(q^{-1}\right)=\frac{\sum_{k=0}^{5} \rho_{k} q^{-k}}{1-q^{-1}},
$$

is selected for control design. Finally, select $W\left(q^{-1}\right)=1$.

Let a pseudo-random-bounded-signal (PRBS) of $N=512$ samples be used to feed the system and the signal-to-noise ratio $\mathrm{SNR}=\operatorname{var}\left[y_{0}(\cdot)\right] / \sigma^{2}=5$. A Monte Carlo simulation over 100 experiments is then performed. The experiments are carried out by considering the same input $u$ and different realizations of the noise $v$.

The Bode diagrams for the closed-loop systems resulting from the standard VRFT formula in (7) are shown in Figure 3. Notice that, as already observed, the method is not statisticaly efficient and the estimator suffers from high variance. Furthermore, in some experiments (i.e., for particular realizations of the noise) the identified controller leads to unstable closed loop system. In these situations, for internal stability to be guaranteed, the (conservative) $\mathcal{H}_{\infty}$ constraint in [23] should be added to the optimization problem.

In this scenario, regularization could help to enhance the statistical performance of the design, by reducing the variance at the price of introducing a small bias. 

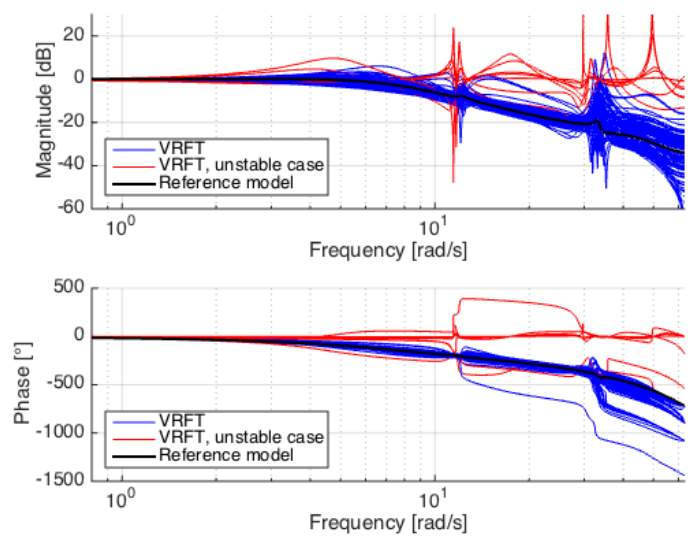

Fig. 3. Frequency responses of the achieved closed-loop systems over 100 experiments: the classical VRFT approach.

First of all, $\Pi$ has to be tuned, whatever its hyperparameterization is. In classical system identification scenario, this is carried out by maximizing the marginal likelihood of the output data (corrupted by the noise) with respect to $\lambda$ and $\alpha$ (empirical Bayes approach). Although controller identification is an errors-in-variable problem (the noise actually affects the input while the output of the controller $u$ is noiseless), it will be shown here that this established technique provides a good tuning of the kernel matrix also in this case (the input noise can be seen - approximately as an equivalent noise on the output).

Let consider different kernels. At each run, the tuning of each $\Pi$ is accomplished by employing the MATLAB ${ }^{\circledR}$ function arxRegul of the System Identification Toolbox (see [16]).

For what concerns the estimate of $\sigma^{2}$, such a parameter is instead selected by using a low bias (i.e., high order) estimate of the plant model $\hat{G}$ :

$$
\hat{\sigma}^{2}=\frac{1}{N} \sum_{t=1}^{N}\left(y(t)-\hat{G}\left(q^{-1}\right) u(t)\right)^{2} .
$$

It should be here remarked that the information given by the estimate of the plant, does not make the design method model-based, since $\hat{G}$ is not directly used to compute the controller but only employed to find the best regularization term. Another way to proceed could be to consider $\sigma^{2}$ as a further hyperparameter.

The final simulation results show that the regularized version of VRFT outperforms the standard approach. In Figure 4, a boxplot with the model reference costs in all the situations is illustrated. In this example, the High Frequency Stable Spline kernel provides the best regularized performance, which is very close to the optimal regularized VRFT in terms of bias, whereas all regularized approaches are better than the classical method in terms of variance.

The additional performance improvement given by the HF kernel is obviously example dependent. However, this result is very important as it shows that, whenever we

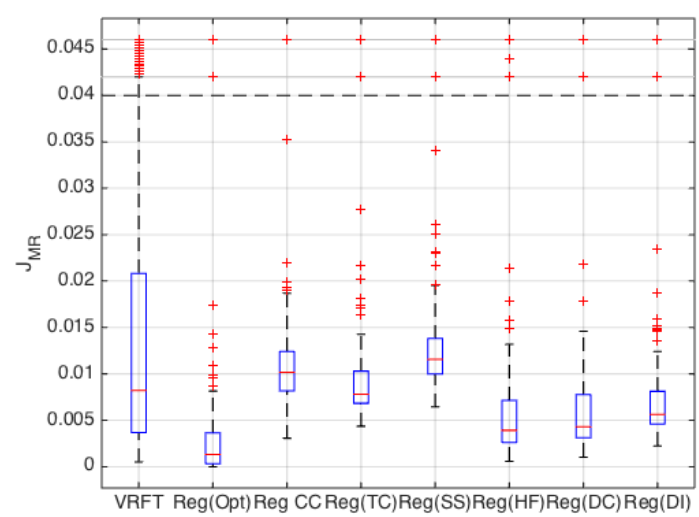

Fig. 4. Closed-loop model matching performance(3) for classical and regularized VRFT (with different kernels).
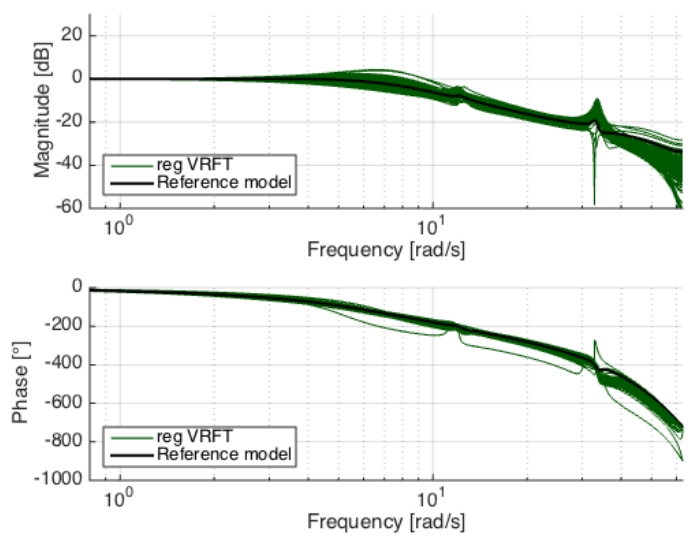

Fig. 5. Frequency responses of the achieved closed-loop systems over 100 experiments: the regularized VRFT approach with the HF kernel.

have some (even rough) information about the plant, such information can be exploited to further improve the statistical performance of the estimate. In this example, it is sufficient to know that the system is resonant and that we expect the optimal control law to properly compensate the output oscillations (because we selected a non-resonant $M$ ). Therefore, HF kernel becomes a good choice as it allows to model the impulse response of an oscillatory system and accurately matches the optimal controller.

The Bode plots of the closed loop system obtained with the HF-regularized identified controller are reported in Figure 5 to be compared with the standard performance of Figure 3.

Finally, the bayesian estimate is compared to the regularized approach in [10]. In Figure 7, it is evident that such an approach is outperformed by the approach proposed here. This can be also appreciated in Figure 4 (the approach in [10] is denoted as Reg CC, standing for the Convex-Concave procedure required to tune the kernel).

Notice that both the above presented regularized approaches are not far from the ideal performance given in Figure 6. 

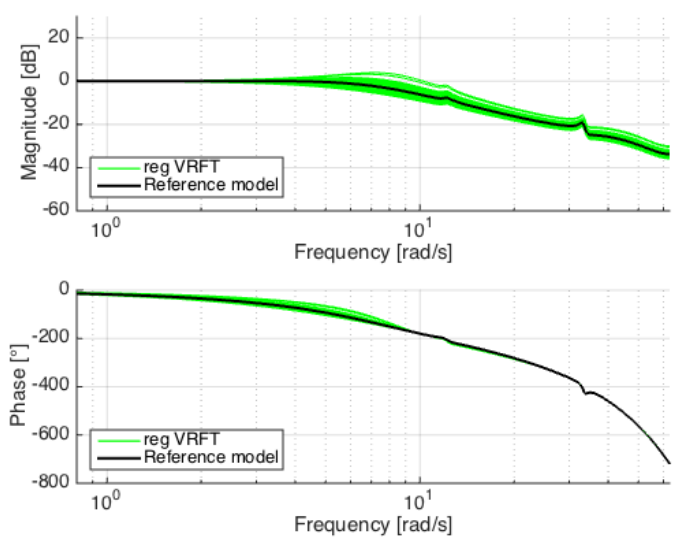

Fig. 6. Frequency responses of the achieved closed-loop systems over 100 experiments: the regularized VRFT approach with the optimal kernel.
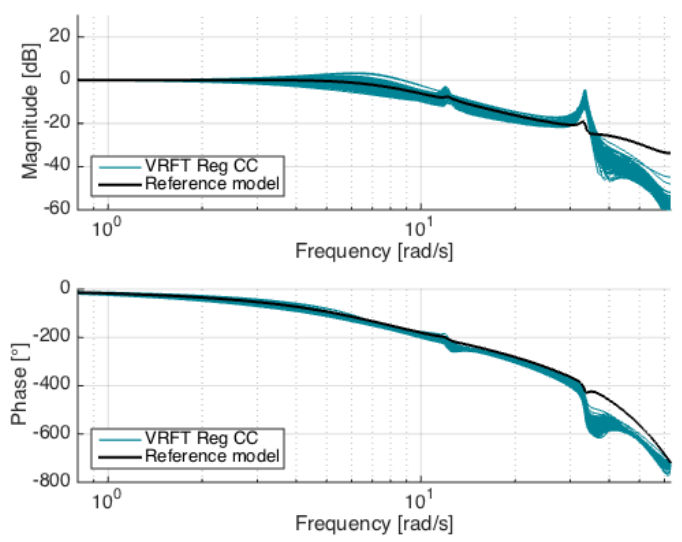

Fig. 7. Frequency responses of the achieved closed-loop systems over 100 experiments: the regularized VRFT approach with the kernel in [10].

\section{CONCLUSIONS}

Regularization is a well-established tool for finding the best bias-variance trade-off in estimation problems. The increase of performance in system identification has been adequately proven and, in particular, the Bayes perspective gave an interesting insight to suitably tune the regularization term.

In this paper, the Bayesian approach to regularization is applied to the VRFT method for direct data-driven design of feedback controllers. Regularization has already been shown to be a useful tool in this context, as VRFT is based on IV estimates, which suffer from high variance. The main results of this paper are two: (i) the optimal regularization term has the same form of that used in system identification given in [5]; (ii) the bayesian approach for regularization may outperform the existing approach for regularized VRFT in [10]. The latter observation holds especially when a-priori knowledge of the system dynamics is available to suitably address the selection of the kernel.

In future work, we will further investigate the use of the marginal likelihood approach in data-driven control design.

\section{REFERENCES}

[1] B.D.O. Anderson and Y. Liu. Controller reduction: concepts and approaches. Automatic Control, IEEE Transactions on, 34(8):802812, 1989.

[2] Marco C. Campi, Andrea Lecchini, and Sergio M. Savaresi. Virtual reference feedback tuning: a direct method for the design of feedback controllers. Automatica, 38(8):1337-1346, 2002.

[3] M.C. Campi and S.M. Savaresi. Direct nonlinear control design: The virtual reference feedback tuning (vrft) approach. IEEE Transactions on Automatic Control, 51(1):14-27, 2006.

[4] Tianshi Chen, Henrik Ohlsson, Graham C Goodwin, and Lennart Ljung. Kernel selection in linear system identification. Part II: A classical perspective. In 50th IEEE Conference on Decision and Control and European Control Conference (CDC-ECC), pages 43264331. IEEE, 2011.

[5] Tianshi Chen, Henrik Ohlsson, and Lennart Ljung. On the estimation of transfer functions, regularizations and gaussian processes - revisited. Automatica, 48(8):1525-1535, 2012.

[6] J.C. Doyle, B.A. Francis, and A. Tannenbaum. Feedback control theory, volume 134. Macmillan New York, 1992.

[7] S. Formentin, M. Corno, SM Savaresi, and L. Del Re. Direct datadriven control of linear time-delay systems. Asian Journal of Control, 2012.

[8] S. Formentin, P. De Filippi, M. Corno, M. Tanelli, and S.M. Savaresi. Data-driven design of braking control systems. IEEE Transactions on Control Systems Technology, (99):1-8, 2012.

[9] S. Formentin and S.M. Savaresi. Virtual Reference Feedback Tuning for linear parameter-varying systems. In IFAC World Congress, Milan, 2011.

[10] Simone Formentin and Alireza Karimi. Enhancing statistical performance of data-driven controller tuning via $\mathcal{L}_{2}$-regularization. Automatica, 50(5):1514-1520, 2014.

[11] G.O. Guardabassi and S.M. Savaresi. Approximate feedback linearization of discrete-time non-linear systems using virtual input direct design. Systems \& Control Letters, 32(2):63-74, 1997.

[12] H. Hjalmarsson. Iterative feedback tuning - an overview. Int J. Adapt. Control Signal Process., 16, 2002.

[13] A. Karimi, L. Miskovic, and D. Bonvin. Iterative correlation-based controller tuning. International Journal of Adaptive Control and Signal Processing, 18(8):645664, 2004.

[14] I.D. Landau, D. Rey, A. Karimi, A. Voda, and A. Franco. A flexible transmission system as a benchmark for robust digital control. European Journal of Control, 1995.

[15] L. Ljung. System identification: theory for the user. Prentice-Hall Englewood Cliffs, NJ, 1987.

[16] Lennart Ljung and Tianshi Chen. What can regularization offer for estimation of dynamical systems? In Adaptation and Learning in Control and Signal Processing, volume 11, pages 1-8, 2013.

[17] G. Pillonetto, A. Chiuso, and G. De Nicolao. Prediction error identification of linear systems: A nonparametric gaussian regression approach. Automatica, 47(2):291-305, 2011.

[18] G. Pillonetto and G. De Nicolao. A new kernel-based approach for linear system identification. Automatica, 46(1):81-93, 2010.

[19] Gianluigi Pillonetto and Giuseppe De Nicolao. Kernel selection in linear system identification. Part I: A gaussian process perspective. In 50th IEEE Conference on Decision and Control and European Control Conference (CDC-ECC), pages 4318-4325. IEEE, 2011.

[20] Gianluigi Pillonetto, Francesco Dinuzzo, Tianshi Chen, Giuseppe De Nicolao, and Lennart Ljung. Kernel methods in system identification, machine learning and function estimation: A survey. Automatica, 50(3):657-682, 2014

[21] T. Soderstrom. Errors-in-variables methods in system identification. Automatica, 43(6):939-958, 2007.

[22] Torsten Söderström and Mei Hong. Identification of dynamic errorsin-variables systems with periodic data. In 16th IFAC World Congress on Automatic Control, Prague, Czech Republic, 2005.

[23] K. van Heusden, A. Karimi, and D. Bonvin. Data-driven model reference control with asymptotically guaranteed stability. International Journal of Adaptive Control and Signal Processing, 2011.

[24] K. van Heusden, A. Karimi, and T. Söderström. On identification methods for direct data-driven controller tuning. International Journal of Adaptive Control and Signal Processing, 25(5):448-465, 2011. 